\title{
Evidence for Mitotic Crossing-over During the Parasexual Cycle of the Cellular Slime Mould Dictyostelium discoideum
}

\author{
By E. B. GINGOLD* AND J. M. ASHWORTH† \\ Biochemistry Department, School of Biological Sciences, Leicester University, \\ Leicester $L E \mathrm{I} 7 R H$
}

(Received 6 May 1974)

\begin{abstract}
SUMMARY
The genes conferring white fruiting bodies (whi), growth-temperature sensitivity in strain $\mathrm{X9}\left(t s g D_{12}\right)$ and resistance to methanol or acriflavine (acrAI) are linked in a group designated chromosome II. A gene, present in strain AX2 and involved in the ability of this strain to grow axenically (axe2), has also been located on chromosome II. An analysis of diploid strains derived from a diploid initially heterozygous for all known genes of chromosome II has revealed that mitotic crossing-over occurs during the vegetative growth of diploid cells. An analysis of the classes of diploid thus isolated leads to a provisional ordering of the genes on chromosome II: centromere, whi, axez, tsg DI 2, acr $A r$. The final element necessary to establish the existence of a parasexual cycle in Dictyostelium discoideum has thus been demonstrated.
\end{abstract}

\section{INTRODUCTION}

The life-cycle of the cellular slime mould Dictyostelium discoideum has features which have made this organism particularly attractive for studies of cell differentiation (for reviews see Bonner, 1967; Garrod \& Ashworth, 1973). The application of genetic methods of analysis to this organism has, however, been delayed by the absence of any well-defined system of recombination. Previous work has shown the existence of both diploid and haploid states and the isolation of one type from the other (Sussman \& Sussman, 1963), but the lack of evidence for a sexual cycle left the nature of these conversions obscure. Loomis \& Ashworth (1968) showed that two haploids carrying independent genes for minute colony size could fuse to give diploids with normal plaque morphology and Sinha \& Ashworth (1969) used this system to investigate the formation and subsequent breakdown of diploids. They were able to demonstrate that most of the elements of a parasexual cycle, similar to that found in other fungi (Pontecorvo, 1956), were present. Fusion of two cells followed by fusion of their nuclei was shown to yield diploids of an unstable nature. It was suggested that these diploids gave rise to haploids via the intermediate formation of aneuploid cells. Recombination of parental markers was clearly seen in these haploid products.

Cytological observations by Brody \& Williams (1974) are in agreement with this interpretation of the nature of the haploidization of diploid strains.

Loomis (1969) improved the techniques for diploid selection by the use of mutant haploid strains carrying alleles conferring temperature sensitivity. When cells of two such strains were mixed at the permissive temperature and the mixture plated at the restrictive temperature, heterozygous diploid clones were obtained. Katz \& Sussman (1972), using this selection

\footnotetext{
* Present address: Genetics Department, School of Biological Sciences, Leicester University.

$\dagger$ Present address: Biology Department, University of Essex, Colchester $\mathrm{CO}_{4} 3 \mathrm{SQ}$.
} 
system, confirmed the parasexual nature of the genetic recombination cycle, but their results differed from those of Sinha \& Ashworth (1969), especially in relation to the stability of the diploid state. Katz \& Sussman (1972) also showed that a number of their genetic markers were linked. This has opened the way for the investigation of the final element required to characterize a parasexual cycle, mitotic crossing-over (Pontecorvo, 1956; Pontecorvo \& Käfer, 1958).

Mitotic crossing-over can result in one or more markers in a diploid changing from a heterozygous to a homozygous state while other markers remain heterozygous. If one of the markers becoming homozygous is recessive, recombinant diploids can be identified by the appearance of this characteristic. Katz \& Sussman (1972) in fact described a number of diploid progeny clones which displayed cycloheximide resistance, a characteristic determined by a recessive allele, whilst still remaining heterozygous at a number of other unlinked loci. They did not, however, postulate mitotic recombination to explain the occurrence of these clones. Instead they suggested that a single chromosome had been lost and the cell then returned to the diplophase by the duplication of the sister chromosome. Such a mechanism would result in homozygosity over the entire chromosome. In contrast, mitotic crossingover results only in that portion of the chromosome distal to the crossover event becoming homozygous, the proximal markers (i.e. those between the exchange and the centromere) and markers on the other arm of the chromosome remaining heterozygous.

We show that many, if not all, of the diploid clones we have isolated and identified as homozygous for a recessive marker are in fact products of mitotic crossover events. Haploids isolated from these diploids display recombination of linked genes.

\section{METHODS}

Chemicals. $N$-methyl- $N^{\prime}$-nitro- $N$-nitrosoguanidine (NTG) was obtained from the Aldrich Chemical Co., Milwaukee, Wisconsin, U.S.A., and cycloheximide and streptomycin sulphate from Sigma.

Strains. The genetic notation used in this paper is described in Table I.

All strains have the same mating type and can be traced to $D$. discoideum NC4 isolated by Raper (1935). Strain AX2 (ATCC24397) is capable of growth in the axenic medium described by Watts \& Ashworth (1970). Strains G8 and G24 are temperature sensitive mutants of AX2, able to grow at 22 but not at $27^{\circ} \mathrm{C}$, and obtained after treatment with NTG as described by Yanagisawa, Loomis \& Sussman, 1967. The genes conferring the temperature sensitivity have been designated $t s g I 4$ and $t s g 24$, respectively. Strain $X 9$ is a haploid which is incapable of growth in axenic medium; it carries the genetic markers $t s g D_{I 2}, c y c A I, a c r A I$, whi, bwn, and was kindly supplied by Drs K. L. Williams and P. C. Newell of Oxford University. It is derived from the diploid DP4 which was formed by fusing strains M28 and NPI4. Strain NPI 4 is an acriflavin (or methanol) resistant mutant (acrAI) of strain TSI 2. Strains M28 and TSI 2 were produced by Katz \& Sussman (I972); strains NPI4, DP4 and X9 were produced by Williams, Kessin \& Newell (1974b).

Diploid formation. The haploid strains to be crossed were generally first grown in association with the bacterium Klebsiella aerogenes on SM agar plates and harvested when the bacterial lawns began to clear but before the aggregation stage (Sussman, 1966). The cells were washed repeatedly by centrifugation to remove most of the remaining bacteria. Portions of the two strains containing $5 \times 10^{6} \mathrm{cells} / \mathrm{ml}$ were mixed in $\mathrm{I} \mathrm{ml}$ of $0.0167 \mathrm{M}$-phosphate buffer $\left(\mathrm{pH}_{5} \cdot 2\right)$. Controls with only one strain at $10^{7}$ cells $/ \mathrm{ml}$ were also set up. The suspensions were incubated with shaking for $\mathrm{I} 5 \mathrm{~h}$ at $22{ }^{\circ} \mathrm{C}$. During this time the cells aggregated 
Table I. Genetic notation

\author{
Character \\ Temperature sensitive growth \\ Cycloheximide resistance \\ Methanol or acriflavine \\ resistance \\ Fruiting body colour \\ Pigment production
}

Ability to grow in axenic medium

\author{
Mutant allele* \\ tsg - unable to grow at $27^{\circ} \mathrm{C}$ \\ $c y c$ - able to grow in the \\ presence of $400 \mu \mathrm{g}$ cyclo- \\ heximide/ml \\ acr $A$ - able to grow in the \\ presence of $2 \%(\mathrm{v} / \mathrm{v})$ methanol \\ whi-white \\ $b w n$ - brown pigment produced \\ by clones 5 days after their \\ becoming visible \\ axe 2 - one of the genes neces- \\ sary for the axenic growth of \\ strain $\mathrm{AX2}$
}

\author{
Wild-type allele $\dagger$ \\ tsg ${ }^{+}$- able to grow at $27{ }^{\circ} \mathrm{C}$ \\ $c y c^{+}$- unable to grow in the \\ presence of $400 \mu \mathrm{g}$ cyclo- \\ heximide $/ \mathrm{ml}$ \\ $\operatorname{acr} A^{+}$- unable to grow in the \\ presence of $2 \%(\mathrm{v} / \mathrm{v})$ methanol \\ whi $i^{+}$yellow \\ $b w n^{+}$- no brown pigment \\ production \\ axe $2^{+}$- unable to grow \\ axenically
}

* $c y c$ has been referred to previously as act (Loomis \& Ashworth, I968; Sinha \& Ashworth, 1969) and $c y$ (Katz \& Sussman, 1972), and whi, bwn and $t s g$ as $w h, b r$ and $t^{\S}$ respectively (Katz \& Sussman, 1972).

$\dagger$ The wild-type allele is in all cases dominant.

into small spheres. These aggregates were then disrupted by the repeated passage of the suspension through the tip of a Pasteur pipette and plated directly on SM agar, in association with $K$. aerogenes. After 4 days' incubation at $27^{\circ} \mathrm{C}$ a number of plaques appeared on the plates from crosses. When tested, these were generally found to be temperature insensitive clones with diploid spore size (Sussman \& Sussman, 1962) and with the phenotype predictable from a diploid genotype. Occasionally a few resistant haploid clones were found, both on control plates and amongst the clones from the crosses, but these were not difficult to distinguish from the diploids. Diploids arose at a frequency of $\mathrm{IO}^{-4}$ to $\mathrm{IO}^{-5}$. This procedure is similar to that described by Williams, Kessin \& Newell (I974a).

Before further analysis the diploids were always taken through at least one clonal passage to eliminate possible contamination with parental cells.

Determination of spore size. Diploid and haploid spores could be consistently distinguished by visual comparison with known spores under a microscope. In addition, some clones were quantitatively compared using a Coulter counter (Sackin \& Ashworth, 1969). These results were always consistent with the visual classifications.

Selection of segregants displaying recessive markers. Spores from diploid clones, sensitive to cycloheximide and methanol but carrying recessive alleles for resistance to these chemicals, were plated in association with $K$. aerogenes on to SM agar $+400 \mu \mathrm{g}$ cycloheximide $/ \mathrm{ml}$ and $\mathrm{SM}$ agar $+2 \%(\mathrm{v} / \mathrm{v})$ methanol at about $10^{4}$ spores/plate. After 3 days' incubation at $22{ }^{\circ} \mathrm{C}$ resistant plaques appeared in the bacterial lawn. Although the frequency of appearance of such clones varied, it was invariably at least 100 times greater than the spontanous mutation frequency. The segregants were examined to determine spore size, and spotted on to bacterial lawns on a series of plates. One plate from the series was incubated at $27{ }^{\circ} \mathrm{C}$ to determine temperature sensitivity. Other plates contained methanol and cycloheximide, and a control plate enabled colour markers to be determined. This method yielded both haploid and diploid segregants.

A second method of haploid selection made use of the recessive genes for axenic growth (see also Williams et al. I974a). A cross between an axenic and a non-axenic strain results in the formation of non-axenic diploids. However, if about $10^{5}$ spores from such a diploid are inoculated into $\mathrm{I} \mathrm{ml}$ axenic media (containing $100 \mu \mathrm{g}$ streptomycin sulphate $/ \mathrm{ml}$ and 86 mM-glucose), axenic haploid segregants appear after about $\mathrm{I}$ week of shaking incubation 
Table 2. Phenotypes of haploid segregants from diploids of the cross $\mathrm{X} 9 \times \mathrm{G} 8$

Resistant segregants were selected by plating on media containing $400 \mu \mathrm{g}$ cycloheximide/ml or $2 \%(\mathrm{v} / \mathrm{v})$ methanol, and haploids separated from diploids by examination of spore size. Brown: non-brown showed no linkage to the other characteristics, both types appearing in most classes. Overall, of 43 clones examined for brown pigment, 23 were brown.

\begin{tabular}{|c|c|c|c|}
\hline \multicolumn{2}{|c|}{ Response to } & \multicolumn{2}{|c|}{ No. of segregants* } \\
\hline Methanol & Cycloheximide & White & Yellow \\
\hline \multicolumn{4}{|c|}{ (I) Selected on methanol } \\
\hline $\mathrm{r}$ & $\mathbf{r}$ & 54 & 0 \\
\hline $\mathrm{r}$ & s & $\begin{array}{c}15 \\
\text { (all Tsg) }\end{array}$ & $\stackrel{2}{\text { (all Tsg) }}$ \\
\hline \multicolumn{4}{|c|}{ (2) Selected on cycloheximide } \\
\hline $\mathrm{r}$ & r & $\begin{array}{l}15 \\
\text { (all Tsg) }\end{array}$ & 0 \\
\hline s & $\mathbf{r}$ & 0 & (all $\stackrel{4}{\mathrm{~T}} \mathrm{sg}^{+}$) \\
\hline
\end{tabular}

at $22{ }^{\circ} \mathrm{C}$. These axenic haploids can be cloned and their phenotypes determined as before.

Tests for brown pigment allele. As the appearance of this pigment is delayed, clones were spotted well spaced on test plates of $K$. aerogenes and scored 5 days after growth first became apparent.

\section{RESULTS}

\section{Demonstration of linkage on chromosome II}

The diploids examined come from either the cross $\mathrm{x} 9$ (tsgDI2 tsgI4 ${ }^{+}$cycAI acrAI whi $b w n) \times \mathrm{G} 8\left(t s g \mathrm{DI2}^{+} \operatorname{tsgI}_{4} \mathrm{cycAI} \mathrm{I}^{+} a c r A I^{+} w h i^{+} b w n^{+}\right)$or $\mathrm{X} 9 \times \mathrm{G} 24$, where $\mathrm{G} 24$ is identical to G8 in all known markers except that tsg24 replaces $t s g$ I4. Strains G8 and G24 also contain an unknown number of alleles enabling these strains to grow in axenic media; these are not present in $\mathrm{x} 9$.

Strain $\mathrm{x} 9$ was chosen for this work as it was known that a number of its markers were linked. Katz \& Sussman (I972) reported linkage of whi to $t \operatorname{sg} D_{I 2}$ (designated $w h^{-}$and $t_{1}^{s}$ respectively) and acr $A I$ has been shown to be linked to these genes by Williams et al. (1974b). This linkage group has been designated chromosome II. The linkage group carrying $c y c A I$, discussed by Katz \& Sussman (I972), has been designated chromosome I.

Table 2 shows the phenotypes of a number of haploids obtained by plating spores from diploid clones of the cross $\mathrm{x} 9 \times \mathrm{G} 8$ on to media containing methanol and cycloheximide. The vast majority of these haploids are of types expected from the known linkages. Thus, for example, methanol resistant haploids were generally white and temperature sensitive. The cycloheximide resistant and brown pigment genes clearly segregated independently from these characteristics. None the less, it can be seen that two haploids showing a nonparental reassortment of chromosome II genes were found. These haploids were methanol resistant and temperature sensitive, but yellow. Back-crossing to $\mathrm{G} 8$ and $\mathrm{x} 9$ showed that these strains carried $\operatorname{tsg} D I 2$. Thus they were of the genotype $\operatorname{tsg} D I 2$ acrAI whi+ $\left(\mathrm{cycAI} \mathrm{I}^{+}\right.$ $\left.b w n^{+}\right)$, a recombination of the genes carried on chromosome II, and represented the first indication of crossing-over.

A marker relating to axenic growth has also been located on chromosome II. All the 


\section{Table 3. Phenotypes of methanol resistant diploids selected from methanol sensitive diploid clones of the cross $\mathrm{X} 9 \times \mathrm{G} 24$}

Methanol resistant clones were isolated as in Table 2 and the diploid clones further characterized. The cross $\times 9 \times$ G24 was used for this analysis as the methanol resistant segregants from this cross yielded a far higher proportion of diploids. The methanol resistant diploids examined came from eight individual sensitive diploid clones. Only a portion of the resistant diploid clones have been tested for ability to yield axenic haploid segregants as it is necessary to test each clone at least three times before it can be scored with certainty as unable to yield such segregants.

$\begin{array}{cclccc}\begin{array}{c}\text { Temperature } \\ \text { sensitivity }\end{array} & \begin{array}{c}\text { No. of } \\ \text { clones }\end{array} & \text { Colour } & \text { No. tested } & \begin{array}{c}\text { No. yielding } \\ \text { axenic haploids }\end{array} & \begin{array}{c}\text { No. not yielding } \\ \text { axenic haploids }\end{array} \\ \begin{array}{c}\text { Insensitive } \\ \text { Sensitive }\end{array} & 7 & \text { All yellow } & 2 & 2 & 0 \\ & 59 & 58 \text { yellow, } & 16 & 13 & 3 \\ \text { I white } & \text { I } & 0 & \text { I }\end{array}$

haploids in Table 2 were found to be non-axenic. However, it was possible to select haploids able to grow axenically by incubating spores from the diploids in axenic media. This was done for a number of diploid clones but in each case, on cloning, all the axenic haploids obtained were found to be temperature sensitive, yellow, and sensitive to methanol and cycloheximide. The only variation between the clones was the presence or absence of brown pigment, about 30 of the roo clones examined producing the pigment. Clones resistant to either methanol or cycloheximide could only be obtained by plating at high concentrations; they could only be obtained at frequencies less than $10^{-5}$ and even then were invariably yellow. Thus it appears that at least one gene affecting axenic growth is located on the chromosome carrying $w i^{+} a c r A I^{+}$. The allele for axenic growth has been provisionally designated axez, with the, presumably dominant, non-axenic allele designated axe $2^{+}$. Williams et al. (1974b) have now also reported an axenic gene on this chromosome.

\section{Characterization of methanol-resistant diploids isolated from sensitive clones}

It has been established that chromosome II carries the four genes: $\operatorname{tsg} D$ acr $A$ whi axe2. However, when the diploids resulting from the crosses $\mathrm{X} 9 \times \mathrm{G} 8$ or $\mathrm{X} 9 \times \mathrm{G} 24$ were plated on methanol-containing media, methanol resistant diploids as well as the methanol resistant haploids discussed previously were obtained. Such clones could clearly be seen to be diploids as their spore sizes were unchanged from those of the original diploid clones when measured with a Coulter counter. In addition, the brown and cycloheximide resistance loci in these diploids were still heterozygous since the recessive alleles could be recovered on haploidization.

If these diploids arose in the manner suggested by Katz \& Sussman (1972) for their cycloheximide resistant diploids, namely a loss and reduplication of a complete chromosome, then all the markers on chromosome II should have become homozygous. The results showed this not to be the case (Table 3). Thus, for example, if whi had become homozygous along with acrAI, the diploids would be expected to be white, yet in all but one case they were yellow. In addition, a duplication of the entire chromosome would result in axe $2^{+}$ becoming homozygous - that is, axe 2 being eliminated from the clone. If this occurred it would not be possible to isolate axenic haploids from such a clone. In most cases such an isolation was indeed possible (Table 3 ) and in fact was no more difficult than with the original diploid clones. Thus these diploids appear to have arisen from mitotic crossing-over, 
Original heterozygote

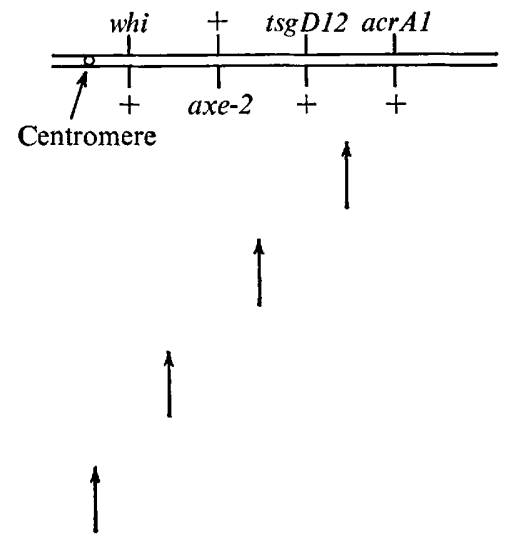

Point of cross-over event

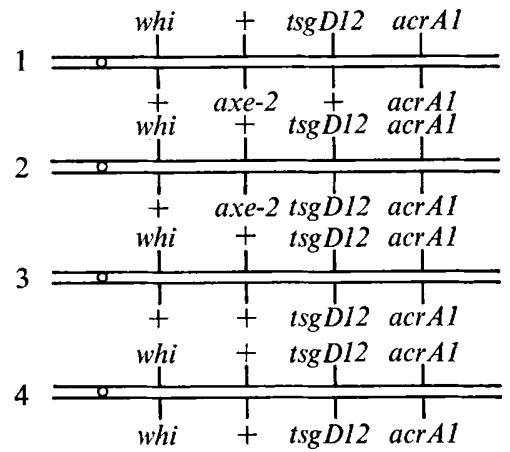

Partial homozygote detected

Fig. I. Origin of the four classes of diploid described in Table 3. The phenotypes of the diploids resulting from the various possible crossover events are: (I) methanol resistant, temperature insensitive, capable of producing axenic haploids and yellow; (2) methanol resistant, temperature sensitive, capable of producing axenic haploids and yellow; (3) methanol resistant, temperature sensitive, incapable of producing axenic haploids and yellow; (4) methanol resistant, temperature sensitive, incapable of producing axenic haploids and white.

resulting in some of the markers on the chromosome becoming homozygous and others remaining unaffected.

Of all the clones listed in Table 3, only one could have arisen from a complete duplication of chromosome II. This diploid was temperature sensitive, white, and unable to produce haploid segregants capable of axenic growth, and had thus become homozygous for all four markers. But even here it is not necessary to invoke complete duplication of a chromosome since a crossover event between the centromere and the most proximal gene would produce such a result.

From the results in Table 3 it is possible to present a provisional order for the four genes and centromere following the method of Pontecorvo \& Käfer (1958). This is based on the assumption that as mitotic crossing-over is rare, double crossovers on the same arm can be ignored. Thus if crossovers are selected using a particular marker, all markers distal to it on the chromosome will always also become homozygous. On the other hand, proximal markers will become homozygous only in some clones. The closer a marker is to the centromere the less likely it is to become homozygous, since it becomes more likely that the crossover event will take place between this marker and the one used for selection than between the former and the centromere. It can be seen from the results that acrAI is, in fact, the most distal marker as no other marker always becomes homozygous with it. The low frequency of clones remaining heterozygous for $t s g D_{I 2}$, i.e. temperature insensitive, indicates that this locus is close to acr $A I$. The colour gene whi, on the other hand, appears to be close to the centromere (and may in fact be on the other arm if the one white clone represents a chromosome duplication). The axez locus appears to be between whi and tsgDI2 since three yellow as well as the one white diploid segregant had lost the ability to yield axenic haploids. The position of axez must, however, remain provisional, as such loss could have occurred in some instances as a result of separate events on other chromosomes bearing axenic genes. (The elimination of any one such gene from a clone is sufficient to cause it to 
lose its ability to yield axenic haploids.) This appears unlikely because of the low frequency of mitotic crossing-over and consequent very low coincidence of two crossovers in the same cell, but separate events cannot be eliminated for every case. If chromosome II itself carries more than one axenic gene this procedure would map the most distal of these genes on the acr $A I$ arm.

As shown in Fig. I, the provisional map of genes on chromosome II consistent with these results is:

centromere whi axe2 tsgDI2 acrAr.

\section{Recombinant haploids isolated from diploids that have undergone mitotic crossing-over}

The final demonstration of the occurrence of mitotic crossing-over in diploids is the isolation from these of haploids showing recombination of linked genes. This is clearly seen in the axenic haploid segregants obtained from these diploids. All these segregants were methanol resistant and yellow - that is, whi ${ }^{+}$axez acr $A I$ - whereas methanol resistant axenic strains could only be obtained from the original diploid clone (in which a crossover event had not occurred) at a frequency of about $\mathrm{IO}^{-5}$. Likewise the recombination of methanol resistance and the yellow allele had also been rare.

A prediction from the model of mitotic crossing-over is that the reciprocal products of crossing-over should be carried by another cell (Pontecorvo, I956). Thus, while some diploids would become homozygous for methanol resistance, other diploids should become homozygous for methanol sensitivity. A practical difficulty is that such diploids cannot be distinguished from the heterozygote. However, such a diploid was in fact isolated from the cross $\mathrm{X} 9 \times \mathrm{G} 24$. It was observed that one clone had a larger plaque size and a deeper yellow spore colour than any of its neighbours. Investigation of this clone left the reasons for this morphological change unclear but revealed that this clone, unlike other diploid clones from the cross, was unable to give rise to methanol resistant haploids. It was, however, still able to give rise to cycloheximide resistant haploids, many of which on investigation were found to be white, and represented a whi acr $A I^{+}$recombinant type. This diploid had clearly become homozygous for $\operatorname{acr} A I^{+}$while remaining heterozygous for $w h i$.

\section{DISCUSSION}

Katz \& Sussman (1972) have carried out studies similar to ours except that they followed the appearance of diploids, homozygous for the recessive allele $c y c A I$ carried on chromosome I, amongst the progeny of heterozygous diploids carrying both $c y c A I$ and $c y c A I^{+}$ alleles. They found that all the diploids homozygous for $c y c A I$ had become, simultaneously, homozygous for two other markers on chromosome I, and suggested a novel mechanism involving chromosome loss and reduplication to account for their results. In this paper we have argued that analogous diploids involving markers on chromosome II are generally the result of crossover events. It appears possible that this is also the case for chromosome $I$ and that the markers followed by Katz \& Sussman (1972) are located distal to cycAI on the chromosome. Evidence for this has now been presented by Williams et al. (1974b). If this is the case such markers would always become homozygous whenever cycAI becomes homozygous, due to crossing-over between $c y c A I$ and the centromere.

It should be made clear that the present work does not set out to prove that all exceptional diploids in $D$. discoideum arise from mitotic crossing-over. In other fungi this is not the case; Käfer (I96r) demonstrated that diploids homozygous for a particular marker may 
arise through either mitotic crossing-over or chromosomal non-disjunction in Aspergillus nidulans. The primary non-disjunctive event leads on division to one nucleus receiving $(2 n+1)$ and the other $(2 n-1)$ chromosomes. The $(2 n+1)$ type is unstable and gives rise to diploid nuclei which will often have a duplication of one chromosome. In D. discoideum, exceptional diploids which show homozygosity for all markers on one chromosome - such as, for example, the white diploid (Table 3) - may have arisen from a non-disjunctive event of this kind. Brody \& Williams (1974) found rare $(2 n+\mathrm{I})$ chromosome figures in diploid cultures of $D$. discoideum. Thus results with $D$. discoideum appear consistent with a mechanism firmly established in $A$. nidulans, and novel schemes of chromosome loss followed by reduplication are unnecessary.

An earlier report (Sinha \& Ashworth, 1969) described 'intermediate' clones which although displaying a recessive characteristic (small plaque size) none the less continued to segregate other markers. It now appears likely that these clones were in fact diploids that had undergone either a mitotic crossing-over event of a chromosomal non-disjunction.

The methods described in this report open the way to constructing a gene map based on mitotic crossover data in $D$. discoideum. It is, however, necessary to show that the ratios of crossover types are quantitatively repeatable from diploids that have arisen from different parents. Such work will need the isolation of markers more easily scored than the axenic genes. The absolute frequency of crossover events does not appear to be constant in different diploids. For example, at least five times the frequency of formation of methanol resistant diploids is found in diploid clones resulting from the cross $\mathrm{X} 9 \times \mathrm{G} 24$ as for $\mathrm{X} 9 \times \mathrm{G} 8$. In the first cross diploids predominate amongst methanol resistant segregants, in the second haploids. None the less, the types of diploids obtained appear similar in both cases.

A final word is necessary about recent reports of a possible sexual cycle in this organism (Clark, Francis \& Eisenberg, 1973; Erdos, Raper \& Vogen, 1973). This cycle is quite distinct from the parasexual cycle described in the present paper; it involves the fusion of two cells of different mating types to form macrocysts. Work on this sexual cycle has been held up by an inability to germinate the macrocysts and thus it has not yet been possible to demonstrate sexual recombination. None the less, even if it proves possible to exploit this system, the parasexual cycle will still be a useful tool in genetic studies of this organism. In species of slime mould for which macrocyst germination has been successful, a number of weeks is necessary for the macrocyst to mature (Nickerson \& Raper, 1973). Thus the parasexual cycle is clearly a faster method of obtaining recombinants. In addition, the low frequency of mitotic recombination means that loci can be efficiently assigned to linkage groups using the parasexual cycle alone and, once so assigned, can be ordered with respect to one another even when so far apart that meiotic recombination analysis would be difficult (Pontecorvo \& Käfer, 1958).

We thank Drs K. L. Williams, R. H. Kessin and P. C. Newell for preprints of their papers and helpful discussions, the Science Research Council for financial assistance, and Mrs Janet Kwasniak and Miss Julie Johnson for technical assistance. E. B. G. was a Beit Memorial research fellow.

\section{REFERENCES}

Bonner, J. T. (1967). The Cellular Slime Molds. Princeton: Princeton University Press.

BRody, T. \& Williams, K. L. (I974). Cytological analysis of the parasexual cycle in Dictyostelium discoideum. Journal of General Microbiology 82, 37I-383.

Clark, M. A., Francis, D. \& Eisenberg, R. (1973). Mating types in cellular slime molds. Biochemical and Biophysical Research Communications 52, 672-678. 
ERdos, G. W., RAPER, K. B. \& VoGEN, L. K. (1973). Mating types and macrocyst formation in Dictyostelium discoideum. Proceedings of the National Academy of Science of the United States of America 70, 1828I 830.

Garrod, D. \& Ashworth, J. M. (1973). Development of the cellular slime mould Dictyostelium discoideum. Symposia of the Society for General Microbiology 23, 407-435.

KÄFER, E. (196I). The processes of spontaneous recombination in vegetative nuclei of Aspergillus nidulans. Genetics 46, 158I-1609.

Katz, E. R. \& Sussman, M. (1972). Parasexual recombination in Dictyostelium discoideum: selection of stable diploid heterozygotes and stable segregants. Proceedings of the National Academy of Sciences of the United States of America 69, 495-497.

Loomis, W. F. (1969). Temperature-sensitive mutants of Dictyostelium discoideum. Journal of Bacteriology $99,65-69$.

Loomis, W. F. \& Ashworth, J. M. (I968). Plaque-size mutants of the cellular slime mould Dictyostelium discoideum. Journal of General Microbiology 53, 18I-186.

Nickerson, A. W. \& RAPER, K. B. (1973). Macrocysts in the life cycle of the Dictyosteliaceae. II. Germination of the macrocysts. American Journal of Botany 60, 247-254.

Pontecorvo, G. (1956). The parasexual cycle in fungi. Annual Review of Microbiology 1o, 393-400.

Pontecorvo, G. \& Käfer, E. (1958). Genetic analysis based on mitotic recombination. Advances in Genetics 9, $71-104$.

RAPER, K. (I935). Dictyostelium discoideum, a new species of slime mould from decaying forest leaves. Journal of Agricultural Research 50, $135-147$.

SACKIN, M. J. \& Ashworth, J. M. (1969). An analysis of the distribution of volumes amongst spores of the cellular slime mould Dictyostelium discoideum. Journal of General Microbiology 59, 275-284.

Sinha, U. \& AshworTh, J. M. (I969). Evidence for the existence of elements of a parasexual cycle in the cellular slime mould Dictyostelium discoideum. Proceedings of the Royal Society B r73, 531-540.

Sussman, M. (1966). Biochemical and genetic methods in the study of cellular slime mould development. In Methods in Cell Physiology, vol. 2, pp. 397-410. Edited by D. Prescott. New York: Academic Press.

Sussman, M. \& Sussman, R. R. (1962). Ploidal inheritance in Dictyostelium discoideum: stable haploid, stable diploid and metastable strains. Journal of General Microbiology 28, 41 7-429.

Sussman, R. R. \& Sussman, M. (1963). Ploidal inheritance in the slime mould Dictyostelium discoideum: haploidization and genetic segregation of diploid strains. Journal of General Microbiology 30, 349-355.

WatTs, D. J. \& Ashworth, J. M. (1970). Growth of myxamoebae of the cellular slime mould Dictyostelium discoideum in axenic culture. Biochemical Journal 119, I7I-I74.

Williams, K. L., Kessin, R. H. \& Newell, P. C. (1974a). Genetics of growth in axenic medium of the cellular slime mould Dictyostelium discoideum. Nature, London 247, 142-143.

Williams, K. L., Kessin, R. H. \& Newell, P. C. (1974b). Parasexual genetics in Dictyostelium discoideum: mitotic analysis of acrifiavin resistance and growth in axenic medium. Journal of General Microbiology 84, 59-69.

Yanagisawa, K., Loomis, W. F. \& Sussman, M. (1967). Developmental regulation of the enzyme UDPgalactose polysaccharide transferase. Experimental Cell Research 46, 329-334. 